\title{
Journal of Mathematics and Computer Science
}

\section{Weighted Čebyšev-Ostrqwski type integral inequalities with power means}

\author{
Al-Taher A. Ghareeb ${ }^{a, *}$, Samir H. Saker ${ }^{b}$, A. M. Ahmed ${ }^{c, d}$ \\ ${ }^{a}$ Department of Basic Science, Faculty of Engineering, Sinai University, El-Arish, Egypt. \\ ${ }^{b}$ Department of Mathematics, Faculty of Science, Mansoura University, Mansoura 35516, Egypt. \\ cMathematics Department, College of Science, Jouf University, Sakaka (2014), Kingdom of Saudi Arabia. \\ ${ }^{d}$ Department of Mathematics, Faculty of Science, Al-Azhar University, Nasr City (11884), Cairo, Egypt.
}

\begin{abstract}
In this paper, we will prove some new weighted Čebyšev-Ostrowski type inequalities with power mean on time scales. The results will be proved by employing the generalized version of Montgomery's identity with weights that will be proved for our purpose. As special cases, we will derive some new weighted discrete inequalities of Čebyšev-Ostrowski type which to the best of the authors' knowledge are essentially new.
\end{abstract}

Keywords: Čebyšev-Ostrowski inequality, integral inequalities, discrete inequalities, Hölder's inequality, time scales.

2020 MSC: 26D15, 34A40, 39A12, 34N05.

(C)2021 All rights reserved.

\section{Introduction}

The significance of inequalities is increasing day by day in mathematical analysis and harmonic analysis. There are many inequalities which have used in investigating and studied complex problems. In fact during the recent years the theory of inequalities has been considered as an incorporative subject between mathematics and other fields. Interested readers are referred to Holder's inequality, Minkovski's inequality, Young's inequality, Wirtinger's inequality, Hardy's inequality, Grûss's inequality, ČebyŠev's inequality and Ostrowski's inequality, for some current advances in the theory of inequalities. In this paper, we are interested in proving some new inequalities of Čebyšev-Ostrowski's type on time scales.

In 1882 Čebyšev [15] proved the inequality

$$
|\mathcal{T}(f, g)| \leqslant \frac{1}{12}(b-a)^{2}\left\|f^{\prime}\right\|_{\infty}\left\|g^{\prime}\right\|_{\infty},
$$

\footnotetext{
*Corresponding author

Email addresses: altaher_ali2008@yahoo.com (Al-Taher A. Ghareeb), shsaker@mans.edu.eg (Samir H. Saker), amaahmed@ju.edu.sa; ahmedelkb@yahoo.com (A. M. Ahmed)
}

doi: $10.22436 /$ jmcs.022.03.01

Received: 2020-05-04 Revised: 2020-05-25 Accepted: 2020-06-15 
where $f, g:[a, b] \longrightarrow \mathbb{R}$ are two absolutely continuous functions, whose first derivative $f^{\prime}$ and $g^{\prime}$ are bounded and the operator $\mathcal{T}(f, g)$ is defined by

$$
\mathcal{T}(f, g)=\frac{1}{b-a} \int_{a}^{b} f(x) g(x) d x-\left(\frac{1}{b-a} \int_{a}^{b} f(x) d x\right)\left(\frac{1}{b-a} \int_{a}^{b} g(x) d x\right),
$$

and the involved integrals exist and $\|\cdot\|_{\infty}$ denotes the norm in $L_{\infty}[a, b]$ defined as $\|p\|_{\infty}=\operatorname{ess} \sup |p(t)|$.

In 1935 Grúss [17] improved the inequality due to Čebyšev and proved that

$$
|\mathcal{T}(\mathrm{f}, \mathrm{g})| \leqslant \frac{1}{4}(\Phi-\varphi)(\Gamma-\gamma)
$$

provided that $f$ and $g$ are two integrable functions on $[a, b]$ satisfying the condition $\varphi \leqslant f(x) \leqslant \Phi$ and $\gamma \leqslant g(x) \leqslant \Gamma$ for all $x \in[a, b]$. The constant $1 / 4$ is the best possible.

In 1938 Ostrowski [21] proved an interesting integral inequality associated with differentiable mappings which is a relationship between the value of a function $f$ on some points in $(a, b)$ and the integration on $[a, b]$ as follows. If $f:[a, b] \longrightarrow \mathbb{R}$ is continuous on $[a, b]$ and differentiable on $(a, b)$ and its derivative $f^{\prime}:(a, b) \longrightarrow \mathbb{R}$ is bounded in $(a, b)$, then for any $t \in[a, b]$, the following inequality holds

$$
\left|f(t)-\frac{1}{b-a} \int_{a}^{b} f(s) d s\right| \leqslant\left[\frac{1}{4}+\left(\frac{t-\frac{a+b}{2}}{b-a}\right)^{2}\right](b-a)\left\|f^{\prime}\right\|_{\infty} .
$$

The inequality is sharp in the sense that the constant $1 / 4$ cannot be replaced by a smaller quantity.

In 1938 Ostrowski gave a very useful formula to estimate the absolute value of derivation of a differentiable function by its integral mean on a bounded interval. In particular in [21], Ostrowski proved his inequality

$$
\left[f(t)-\frac{1}{b-a} \int_{a}^{b} f(\eta) d \eta\right] \leqslant\left[\frac{(t-a)^{2}+(b-t)^{2}}{2(b-a)}\right] \sup _{\eta \in(a, b)}\left|f^{\prime}(\eta)\right|,
$$

by employing the Montgomery identity. In the last decades Ostrowski's inequality has been generalized and extended by several authors. In the following, we indicate to some of the results in the literature to show the motivation of our aim in this paper.

In 2007 Pachpatte [22] (Theorems 1 and 2) established new generalizations of Ostrowski inequality for two functions, whose derivatives belong to $L^{p}$-spaces for $1 \leqslant p<\infty$. At the same year Rafiq et al. [23] proved some weighted Čebyšev-Ostrowski type integral inequalities containing functions with first derivatives belong to $\mathrm{L}_{\infty}[\mathrm{a}, \mathrm{b}]$.

In 2009 Ahmad et. al. [4] established weighted Čebyšev-Ostrowski type integral inequalities containing functions with first derivatives belong to $L^{p}[a, b]$, for $1 \leqslant p<\infty$ with a norm $\|f\|_{p}=\left(\int_{a}^{b}|f(x)|^{p} d x\right)^{1 / p}$. The Ostrowski type inequality has powerful applications in numerical integration, probability and optimization theory, stochastic, statistics, information and integral operator theory, see for example $[6,7,16$, 20].

In the last few decades numerous authors have been interested in establishing the corresponding discrete analogues of inequalities in various fields of analysis, and as a result, this subject became topic of ongoing research. So it is natural to ask if is it possible to study the bounds of the discrete operator

$$
\mathcal{M}(f, g)=\frac{1}{N+1-a} \sum_{n=a}^{N} f(n) g(n)-\left(\frac{1}{(N+1-a)} \sum_{n=a}^{N} f(n)\right)\left(\frac{1}{(N+1-a)} \sum_{n=a}^{N} g(n)\right),
$$

on the discrete spaces $l^{p}[a, N]$ and $l_{\infty}[a, N]$, where $[a, N] \subset \mathbb{Z}^{+}=\{0,1,2, \ldots\}$ ? The crucial reason for this upsurge of interest in discrete case is due to the fact that discrete operators may even behave differently 
from their continuous counterparts. But the main challenge in establishing discrete analogues is that there are no general methods to study these questions. Therefore, these methods have to be developed starting from the basic definitions. In some cases, it is possible, almost straightforward, to translate or adapt the objects and results from the continuous setting to the discrete setting or vice versa.

However, in some other cases this problem is far from being trivial bounds for discrete analogues of more complicated operators such as singular and fractional operators, maximal Radon transforms (involving integration over a submanifold, or family of submanifolds), are not implied by the corresponding continuous results, and moreover, they are resistant to conventional methods and this returned to the lake of the calculus on the discrete spaces where there is no power rules and also there is no chain rule which are the main tools used in the proofs of the continuous case. To overcame these problems, we will study the boundedness of a generalized weighted power mean operator of the form

$$
\begin{aligned}
\mathcal{H}(f, g)= & \frac{1}{m(a, b)} \int_{a}^{b} w(t) f^{\gamma}(t) g^{\gamma}(t) \Delta t-\frac{1}{m^{2}(a, b)}\left(\int_{a}^{b} w(t) f^{\gamma}(t) \Delta t\right)\left(\int_{a}^{b} w(t) g^{\gamma}(\sigma(t)) \Delta t\right) \\
& -\frac{1}{m^{2}(a, b)}\left(\int_{a}^{b} w(t) g^{\gamma}(t) \Delta t\right)\left(\int_{a}^{b} w(t) f^{\gamma}(\sigma(t)) \Delta t\right) \\
& +\frac{1}{m^{3}(a, b)}\left(\int_{a}^{b} w(t) g^{\gamma}(\sigma(t)) \Delta t\right)\left(\int_{a}^{b} w(t) f^{\gamma}(\sigma(t)) \Delta t\right),
\end{aligned}
$$

on time scales where the measure $m(a, b)$ is defined by $m(a, b)=\int_{a}^{b} w(x) \Delta x$. The general idea is to prove a result for a dynamic inequality where the domain of the unknown function is a so-called time scale $\mathbb{T}$, which may be an arbitrary closed subset of the real numbers $\mathbb{R}$, to avoid proving results twice, once for differential inequality and once again for difference inequality. This idea goes back to its founder Stefan Hilger [18] who started the study of dynamic equations on time scales. Since the integral and discrete inequalities are important in the analysis of qualitative properties of solutions of differential and difference equations, we also believe that the dynamic Hardy type inequalities with weights on time scales will play the same effective role in the analysis of qualitative properties of dynamic equations with boundary conditions like oscillation, nonoscillation and distribution of zeros of solutions. For related dynamic inequalities on time scales, we refer the reader to the papers [8, 9, 24-33] and the books [2, 3].

In recent decades some authors established some weighted Čebyšev type inequalities, Grüss inequality, Ostrowski inequalities on time scales, we refer the reader to the papers $[1,5,10,11]$ and the references they are cited. Since Montgomery 's identity and its extensions have been tools that have been used to prove most of the inequalities of Čebyšev-Ostrowski, it is natural to prove on time scales. In fact in [11] Bohner et al. generalized Montgomery identity and proved that

$$
f(t)=\frac{1}{b-a}\left[\int_{a}^{b} f^{\sigma}(\eta) \Delta \eta+\int_{a}^{b} \Psi(t, \eta) f^{\Delta}(\eta) \Delta \eta\right],
$$

where $a, b \in \mathbb{T}$ with $a<b$ and $f \in C_{r d}^{1}\left([a, b]_{\mathbb{T}}, \mathbb{R}\right)$ and $\Psi:[a, b]_{\mathbb{T}}^{2} \rightarrow \mathbb{R}$ is defined by

$$
\Psi(t, s):= \begin{cases}s-a, & s \in[a, t)_{\mathbb{T}} \\ s-b, & s \in[t, b]_{\mathbb{T}}\end{cases}
$$

for $s, t \in[a, b]_{\mathbb{T}}$. Let $a, b \in \mathbb{T}$ with $a<b$ and $f \in C_{r d}^{1}\left([a, b]_{\mathbb{T}}, \mathbb{R}\right)$. By using the identity (1.3) Bohner et al. proved Ostrowski inequality

$$
\left[f(t)-\frac{1}{b-a} \int_{a}^{b} f^{\sigma}(\eta) \Delta \eta\right] \leqslant\left(\sup _{\eta \in(a, b)}\left|f^{\Delta}(\eta)\right|\right)\left[\frac{h_{2}(t, a)+h_{2}(t, b)}{b-a}\right],
$$


on time scales, where $h_{2}(t, s)$ is the second-order generalized polynomial on time scales and will be defined in the next section.

In 2011 Bohner et al. [12] established weighted Ostrowski-Grüss type integral inequalities on time scales involving functions with first derivatives belonging to $L_{\Delta}^{\infty}(a, b)$ and $L_{\Delta}^{p}(a, b)$ (will be defined in the next section). In particular, they proved that if $f, g \in C_{r d}^{1}\left([a, b]_{\mathbb{T}}, \mathbb{R}\right)$ such that $f^{\Delta}, g^{\Delta} \in L_{\Delta}^{\infty}(a, b)$ then for all $t \in[a, b]_{\mathbb{T}}$, we have

$$
\begin{gathered}
\mid f(t) g(t)-\frac{1}{m(a, b)}\left[g(t) \int_{a}^{b} w(s) f(\sigma(s)) \Delta s+f(t) \int_{a}^{b} w(s) g(\sigma(s)) \Delta s\right] \\
\quad+\left(\frac{1}{m(a, b)} \int_{a}^{b} w(s) f(\sigma(s)) \Delta s\right)\left(\frac{1}{m(a, b)} \int_{a}^{b} w(s) g(\sigma(s)) \Delta s\right) \mid \\
\quad \leqslant\left(\frac{1}{m(a, b)} \int_{a}^{b}(\sigma(s)-t) w(s) \operatorname{sgn}(s-t) \Delta s\right)^{2}\left\|f^{\Delta}\right\|_{\infty}\left\|g^{\Delta}\right\|_{\infty} .
\end{gathered}
$$

where $\mathrm{a}, \mathrm{b} \in \mathbb{T}$ such that $\mathrm{a}<\mathrm{b}$ and $w:[\mathrm{a}, \mathrm{b}]_{\mathbb{T}} \rightarrow[0, \infty)$ and $\mathrm{m}(\mathrm{a}, \mathrm{b})=\int_{\mathrm{a}}^{\mathrm{b}} w(\mathrm{x}) \Delta \mathrm{x}$.

In 2016 Tuna and Liu [34] established new weighted Čebyšev Ostrowski type integral inequalities on time scales in $L^{\infty}$-norm and $L^{p}$-norm, involving function with first derivatives belonging to $L_{\Delta}^{\infty}(a, b)$ and $\mathrm{L}_{\Delta}^{\mathrm{p}}(\mathrm{a}, \mathrm{b})$. They proved that if $\mathrm{f}, \mathrm{g} \in \mathrm{C}_{\mathrm{rd}}^{1}\left([\mathrm{a}, \mathrm{b}]_{\mathbb{T}}, \mathbb{R}\right)$ such that $\mathrm{f}^{\Delta}, \mathrm{g}^{\Delta} \in \mathrm{L}_{\Delta}^{\infty}(\mathrm{a}, \mathrm{b})$, then for all $\mathrm{t} \in[\mathrm{a}, \mathrm{b}]_{\mathbb{T}}$, we have

$$
\begin{aligned}
& \mid \frac{1}{m(a, b)} \int_{a}^{b} w(t) f(t) g(t) \Delta t-\frac{1}{2 m^{2}(a, b)}\left(\int_{a}^{b} w(t) g(t) \Delta t\right)\left(\int_{a}^{b} w(t) f(\sigma(t)) \Delta t\right) \\
& \quad-\frac{1}{2 m^{2}(a, b)}\left(\int_{a}^{b} w(t) f(t) \Delta t\right)\left(\int_{a}^{b} w(t) g(\sigma(t)) \Delta t\right) \mid \\
& \leqslant \frac{1}{2 m^{2}(a, b)} \int_{a}^{b} w(x)[|g(x)| M+|f(x)| N]\left(\int_{a}^{b} \operatorname{sgn}(t-x)(\sigma(t)-x) w(x) \Delta t\right) \Delta x,
\end{aligned}
$$

where $w:[a, b]_{\mathbb{T}} \rightarrow[0, \infty), M=\sup _{a<t<b}\left|f^{\Delta}(t)\right|<\infty$ and $N=\sup _{a<t<b}\left|g^{\Delta}(t)\right|<\infty$.

Following this trend and to develop the study of dynamic inequalities on time scales, we will prove some new weighted Čebyšev-Ostrowski type integral inequalities which contain operators with power means of that is defined by (1.2). To obtain the main results, we will prove a generalized version of Montgomery identity with weights. As special cases, we derive some discrete inequalities which to the best of our knowledge are essentially new. The paper is organized as follows. In Section 2, we present some basic definitions concerning the delta calculus on that we will use in this article. In Section 3, we will prove the main results and derive the special cases.

\section{Preliminaries}

In this section, we present some basic definitions and results concerning the delta calculus on time scales; for more details we refer the reader to the book [13]. A time scale $\mathbb{T}$ is an arbitrary nonempty closed subset of the real numbers $\mathbb{R}$.

Definition 2.1. The forward jump operator and the backward jump operator are defined by $\sigma(t):=\inf \{s \in$ $\mathbb{T}: s>t\}$, and $\rho(t):=\sup \{s \in \mathbb{T}: s<t\}$, where $\sup \emptyset=\inf \mathbb{T}$. A point $t \in \mathbb{T}$, is said to be left-dense if $\rho(t)=t$ and $t>\inf \mathbb{T}$, is right-dense if $\sigma(t)=t$, is left-scattered if $\rho(t)<t$ and right-scattered if $\sigma(t)>t$.

Definition 2.2. A function $f: \mathbb{T} \rightarrow \mathbb{R}$ is said to be right-dense continuous (rd-continuous) provided $f$ is continuous at right-dense points and at left-dense points in $\mathbb{T}$, left hand limits exist and are finite. 
Definition 2.3. For a function $f: \mathbb{T} \rightarrow \mathbb{R}$ we define the derivative $f^{\Delta}(t)$ to be the number, if one exists, such that for all $\epsilon>0$, there is a neighborhood $U$ of $t$ such that for all $s \in U$,

$$
\left|f(\sigma(t))-f(s)-f^{\Delta}(t)(\sigma(t)-s)\right| \leqslant \epsilon|\sigma(t)-s| .
$$

We say that $f$ is delta differentiable on $\mathbb{T}$ provided that $f^{\Delta}(t)$ exists for all $t \in \mathbb{T}$.

The set of all such rd-continuous functions is denoted by $C_{r d}(\mathbb{T})$. Also, the set of functions that are differentiable and whose derivative is rd-continuous is denoted by $C_{r d}^{1}(\mathbb{T})=C_{r d}^{1}(\mathbb{T}, \mathbb{R})$. The graininess function $\mu$ for a time scale $\mathbb{T}$ is defined by $\mu(t):=\sigma(t)-t$, and for any function $f: \mathbb{T} \rightarrow \mathbb{R}$ the notation $f^{\sigma}(t)$ denotes $f(\sigma(t))$.

Without loss of generality, we assume that $\sup \mathbb{T}=\infty$, and define the time scale interval $[a, b]_{\mathbb{T}}$ by $[a, b]_{\mathbb{T}}:=[a, b] \cap \mathbb{T}$. Recall of the following product and quotient rules for the derivative of the product $f g$ and the quotient $f / g$ (where $\mathrm{gg}^{\sigma} \neq 0$, here $g^{\sigma}=g \circ \sigma$ ) of two differentiable functions $f$ and $g$

$$
(\mathrm{fg})^{\Delta}=\mathrm{f}^{\Delta} \mathrm{g}+\mathrm{f}^{\sigma} \mathrm{g}^{\Delta}=\mathrm{fg}^{\Delta}+\mathrm{f}^{\Delta} \mathrm{g}^{\sigma}, \text { and }\left(\frac{\mathrm{f}}{\mathrm{g}}\right)^{\Delta}=\frac{\mathrm{f}^{\Delta} \mathrm{g}-\mathrm{fg} \mathrm{g}^{\Delta}}{\mathrm{g} \mathrm{g}^{\sigma}}
$$

The first chain rule that we will use in this paper is

$$
\left(f^{\gamma}(t)\right)^{\Delta}=\gamma \int_{0}^{1}\left[h f^{\sigma}+(1-h) f\right]^{\gamma-1} \operatorname{dhf}^{\Delta}(t), \quad \gamma \in \mathbb{R},
$$

which is a simple consequence of Keller's chain rule [13, Theorem 1.90]. The second chain rule that we will use in this paper is given in the following. Let $f: \mathbb{R} \rightarrow \mathbb{R}$ be continuously differentiable and suppose $g: \mathbb{T} \rightarrow \mathbb{R}$ is delta differentiable, then $f \circ g: \mathbb{T} \rightarrow \mathbb{R}$ is delta differentiable and

$$
f^{\Delta}(g(t))=f^{\prime}(g(d)) g^{\Delta}(t), \quad \text { for } \quad d \in[t, \sigma(t)] .
$$

In this paper we will refer to the (delta) integral which we can define as follows. If $F^{\Delta}(t)=f(t)$, then the Cauchy (delta) integral of $f$ is defined by $\int_{t_{0}}^{t} f(s) \Delta s:=F(t)-F\left(t_{0}\right)$. It can be shown (see [13]) that if $f \in C_{r d}(\mathbb{T})$, then the Cauchy integral $F(t):=\int_{t_{0}}^{t} f(s) \Delta s$ exists, $t_{0} \in \mathbb{T}$, and satisfies $F^{\Delta}(t)=f(t), t \in \mathbb{T}$. An infinite integral is defined as $\int_{a}^{\infty} f(t) \Delta t=\lim _{b \rightarrow \infty} \int_{a}^{b} f(t) \Delta t$. Integration on discrete time scales is defined by

$$
\int_{a}^{b} f(t) \Delta t=\sum_{t \in[a, b)} \mu(t) f(t) .
$$

The integration by parts formula on time scales reads

$$
\int_{a}^{b} u(t) v^{\Delta}(t) \Delta t=[u(t) v(t)]_{a}^{b}-\int_{a}^{b} u^{\Delta}(t) v^{\sigma}(t) \Delta t
$$

Hölder's inequality states that for $f, g \in C_{r d}\left([a, b]_{\mathbb{T}}, \mathbb{R}\right)$, we have

$$
\int_{a}^{b}|f(t) g(t)| \Delta t \leqslant\left[\int_{a}^{b}|f(t)|^{p} \Delta t\right]^{1 / p}\left[\int_{a}^{b}|g(t)|^{q} \Delta t\right]^{1 / q}
$$

where $p>1,1 / p+1 / q=1$ and $a, b \in \mathbb{T}$. This inequality is reversed if $0<p<1$ and $\int_{a}^{b}|g(t)|^{q} \Delta t>0$, and it is also reversed if $p<0$ and $\int_{a}^{b}|f(t)|^{p} \Delta t>0$.

Now, we define the Taylor monomials defined in [14]. These types of monomials are important because they are intimately related to Cauchy functions for certain dynamic equations which are important in variations of constants formulas. 
Definition 2.4. The Taylor monomials $h_{k}: \mathbb{T} \times \mathbb{T} \rightarrow \mathbb{R}, k \in \mathbb{N}_{0}=\mathbb{N} \cup\{0\}$, are defined recursively as follows. The function $h_{0}$ is defined by $h_{0}(t, s)=1$, for all $s, t \in \mathbb{T}$, and given $h_{k}$ for $k \in \mathbb{N}_{0}$, the function $h_{k+1}$ is defined by

$$
h_{k+1}(t, s)=\int_{s}^{t} h_{k}(\tau, s) \Delta \tau, \text { for all } s, t \in \mathbb{T} .
$$

If we let $h_{k}^{\Delta}(t, s)$ denote for each fixed $s \in \mathbb{T}$, the derivative of $h(t, s)$ with respect to $t$, then

$$
h_{k}^{\Delta}(t, s)=h_{k-1}(t, s), \quad k \in \mathbb{N}, t \in \mathbb{T},
$$

for each fixed $s \in \mathbb{T}$.

The above definition obviously implies $h_{1}(t, s)=t-s$, for all $s, t \in \mathbb{T}$. In the case when $\mathbb{T}=\mathbb{R}$, then $\sigma(t)=t, \mu(t)=0, y^{\Delta}(t)=y^{\prime}(t)$, and

$$
h_{k}(t, s)=\frac{(t-s)^{k}}{k !}, \text { for all } s, t \in \mathbb{R},
$$

for all $s, t \in \mathbb{T}$.

Now, we present the definition of $\Delta$-measurable functions on time scales and we set out the method used in [14, Chapter 5] by Bohner and Guseinov to define the Lebesgue $\Delta$-measure on $\mathbb{T}$. First, by defining the measure $m$ which assigns to each interval $[a, b) \cap \mathbb{T}$ its length, that is $m([a, b))=b-a$. Using $m$, they generate the outer measure $m_{1}^{*}$, defined for each subset $E$ of $\mathbb{T}$ as

$$
m_{1}^{*}(E)= \begin{cases}\inf _{\mathcal{K}}\left\{\sum_{i \in I_{\mathcal{R}}}\left(b_{i}-a_{i}\right)\right\} \in \mathbb{R}^{+}, & \text {if } b \notin E, \\ +\infty, & \text { if } b \in E,\end{cases}
$$

with

$$
\mathcal{R}=\left\{\left\{\left[a_{i}, b_{i}\right) \cap \mathbb{T}\right\}_{i \in I_{\mathcal{R}}}: I_{\mathcal{R}} \subset \mathbb{N}, E \subset \bigcup_{i \in I_{\mathscr{R}}}\left(\left[a_{i}, b_{i}\right) \cap \mathbb{T}\right)\right\} .
$$

A set $A \subset \mathbb{T}$ is said to be $\Delta$-measurable if the following equality

$$
m_{1}^{*}(E)=m_{1}^{*}(E \cap A)+m_{1}^{*}(E \cap(\mathbb{T} \backslash A)),
$$

holds for all subsets $E \subset \mathbb{T}$.

Definition 2.5. We say that $f: \mathbb{T} \rightarrow \mathbb{R}$ is $\Delta$-measurable if for every $\alpha \in \mathbb{R}$, the set

$$
\mathrm{f}^{-1}([-\infty, \alpha))=\{\mathrm{t} \in \mathbb{T}: \mathrm{f}(\mathrm{t})<\alpha\},
$$

is $\Delta$-measurable.

Definition 2.6. Let $E \subset \mathbb{T}$ be a $\Delta$-measurable set and let $p \geqslant 1$ and let $f: E \rightarrow \mathbb{R}$ be a $\Delta$-measurable function. We say that $f$ belongs to $L_{\Delta}^{p}(E)$ provided that either

$$
\int_{E}|f|^{p} \Delta s<\infty, \quad \text { if } 1 \leqslant p<\infty,
$$

or there exists a constant $C \in \mathbb{R}$ such that

$$
|\mathrm{f}| \leqslant \mathrm{C}, \quad \text { if } \mathrm{p}=+\infty,
$$

where $f$ is $\Delta$-almost every where on $E$.

In order to prove our main results in Section 3, we need the following lemmas. 
Lemma 2.7. Assume $\mathrm{F}: \mathbb{T} \rightarrow \mathbb{R}$ is differentiable and positive. If $\mathrm{F}^{\Delta}$ is always positive, then

$$
\left(\mathrm{F}^{\lambda}\right)^{\Delta} \geqslant \mathrm{F}^{\Delta}\left(\mathrm{F}^{\sigma}(\mathrm{t})\right)^{\lambda-1} \text {, if } \lambda \geqslant 1
$$

and

$$
\left(F^{\lambda}\right)^{\Delta} \leqslant F^{\Delta}\left(F^{\sigma}(t)\right)^{\lambda-1} \text {, if } 0 \leqslant \lambda \leqslant 1 .
$$

Proof. If $\mathrm{F}$ is increasing and $\lambda \geqslant 1$, then $\mathrm{F}^{\lambda-1}$ is increasing and thus $\left(\mathrm{F}^{\lambda-1}\right)^{\Delta}>0$ so that

$$
\left(F^{\lambda}\right)^{\Delta}=\left(F F^{\lambda-1}\right)^{\Delta}=F^{\Delta}\left(F^{\sigma}(t)\right)^{\lambda-1}+F\left(F^{\lambda-1}\right)^{\Delta} \geqslant 0 .
$$

This shows (2.2), and (2.3) follows similarly. The proof is complete.

\section{Main results}

Throughout this paper, we assume that the functions (without mentioning) are rd-continuous nonnegative and $\Delta$-differentiable functions, locally $\Delta$-integrable on $[a, b)_{\mathbb{T}}$. We also assume that all the constants and the boundaries of the integrals that appear in the inequalities are real numbers greater than or equal to zero. Let $\mathrm{a}, \mathrm{b} \in \mathbb{T}$ with $\mathrm{a}<\mathrm{b}$ and $\mathrm{f}, \mathrm{g} \in \mathrm{C}_{\mathrm{rd}}^{1}\left([\mathrm{a}, \mathrm{b}]_{\mathbb{T}}, \mathbb{R}\right)$ and $w \in \mathrm{C}_{\mathrm{rd}}^{1}\left([\mathrm{a}, \mathrm{b}]_{\mathbb{T}}, \mathbb{R}^{+}\right)$be non-negative and integrable. Define

$$
p_{w}(x, t)= \begin{cases}\int_{a}^{t} w(\tau) \Delta \tau, & a \leqslant t<x \\ \int_{b}^{t} w(\tau) \Delta \tau, & x<t \leqslant b\end{cases}
$$

and $m(a, b)=\int_{a}^{b} w(x) d x$.

Theorem 3.1. Assume that $M=\sup _{\mathbf{a}<\mathrm{t}<\mathbf{b}}\left|\left(f^{\gamma}(\mathrm{t})\right)^{\Delta}\right|<\infty, N=\sup _{\mathbf{a}<\mathrm{t}<\mathbf{b}}\left|\left(g^{\gamma}(\mathrm{t})\right)^{\Delta}\right|<\infty$ and let $\mathrm{K}=\mathrm{MN}$. Then we have

$$
|\mathcal{H}(f, g)| \leqslant K \int_{a}^{b} \frac{w(x)}{m(a, b)}\left(\frac{1}{m(a, b)} \int_{a}^{b}\left|p_{w}(x, t)\right| \Delta t\right)^{2} \Delta x,
$$

where $\mathcal{H}(\mathrm{f}, \mathrm{g})$ is defined as in (1.2) and $\mathrm{K}>0$ is a psoitive constant.

Proof. By using integration by parts (2.1), we have that

$$
\begin{aligned}
\int_{a}^{b} p_{w}(x, t)\left(f^{\gamma}(t)\right)^{\Delta} \Delta t= & \int_{a}^{x}\left(\int_{a}^{t} w(\tau) \Delta \tau\right)\left(f^{\gamma}(t)\right)^{\Delta} \Delta t+\int_{x}^{b}\left(\int_{b}^{t} w(\tau) \Delta \tau\right)\left(f^{\gamma}(t)\right)^{\Delta} \Delta t \\
= & f^{\gamma}(x) \int_{a}^{x} w(\tau) \Delta \tau-f^{\gamma}(a) \int_{a}^{a} w(\tau) \Delta \tau-\int_{a}^{x} w(t) f^{\gamma}(\sigma(t)) \Delta t \\
& +f^{\gamma}(b) \int_{b}^{b} w(\tau) \Delta \tau-f^{\gamma}(x) \int_{b}^{x} w(\tau) \Delta \tau-\int_{x}^{b} w(t) f^{\gamma}(\sigma(t)) \Delta t \\
= & f^{\gamma}(x)\left(\int_{a}^{x} w(\tau) \Delta \tau+\int_{x}^{b} w(\tau) \Delta \tau\right)-\left(\int_{a}^{x} w(t) f^{\gamma}(\sigma(t)) \Delta t+\int_{x}^{b} w(t) f^{\gamma}(\sigma(t)) \Delta t\right) \\
= & f^{\gamma}(x) \int_{a}^{b} w(t) \Delta t-\int_{a}^{b} w(t) f^{\gamma}(\sigma(t)) \Delta t \\
= & f^{\gamma}(x) m(a, b)-\int_{a}^{b} w(t) f^{\gamma}(\sigma(t)) \Delta t,
\end{aligned}
$$

and thus we obtain the generalized Montgomery identity

$$
f^{\gamma}(x)-\frac{1}{m(a, b)} \int_{a}^{b} w(t) f^{\gamma}(\sigma(t)) \Delta t=\frac{1}{m(a, b)} \int_{a}^{b} p_{w}(x, t)\left(f^{\gamma}(t)\right)^{\Delta} \Delta t .
$$


A. A. Ghareeb, S. H. Saker, A. M. Ahmed, J. Math. Computer Sci., 22 (2021), 189-203

196

Replacing $f$ by $g$ in (3.2), we obtain

$$
g^{\gamma}(x)-\frac{1}{m(a, b)} \int_{a}^{b} w(t) g^{\gamma}(\sigma(t)) \Delta t=\frac{1}{m(a, b)} \int_{a}^{b} p_{w}(x, t)\left(g^{\gamma}(t)\right)^{\Delta} \Delta t .
$$

Now, multiplying both sides of (3.2) and (3.3), we obtain

$$
\begin{gathered}
f^{\gamma}(x) g^{\gamma}(x)-\frac{f^{\gamma}(x)}{m(a, b)} \int_{a}^{b} w(t) g^{\gamma}(\sigma(t)) \Delta t-\frac{g^{\gamma}(x)}{m(a, b)} \int_{a}^{b} w(t) f^{\gamma}(\sigma(t)) \Delta t \\
\quad+\frac{1}{m^{2}(a, b)}\left(\int_{a}^{b} w(t) f^{\gamma}(\sigma(t)) \Delta t\right)\left(\int_{a}^{b} w(t) g^{\gamma}(\sigma(t)) \Delta t\right) \\
=\frac{1}{m^{2}(a, b)}\left(\int_{a}^{b} p_{w}(x, t)\left(f^{\gamma}(t)\right)^{\Delta} \Delta t\right)\left(\int_{a}^{b} p_{w}(x, t)\left(g^{\gamma}(t)\right)^{\Delta} \Delta t\right) .
\end{gathered}
$$

Multiplying equation (3.4) with $w(x) / m(a, b)$ and integrating, and taking absolute values, we get that

$$
\begin{aligned}
& \mid \frac{1}{m(a, b)} \int_{a}^{b} w(t) f^{\gamma}(t) g^{\gamma}(t) \Delta t-\frac{1}{m^{2}(a, b)}\left(\int_{a}^{b} w(t) f^{\gamma}(t) \Delta t\right)\left(\int_{a}^{b} w(t) g^{\gamma}(\sigma(t)) \Delta t\right) \\
& \quad-\frac{1}{m^{2}(a, b)}\left(\int_{a}^{b} w(t) g^{\gamma}(t) \Delta t\right)\left(\int_{a}^{b} w(t) f^{\gamma}(\sigma(t)) \Delta t\right) \\
& \quad+\frac{1}{m^{3}(a, b)}\left(\int_{a}^{b} w(t) g^{\gamma}(\sigma(t)) \Delta t\right)\left(\int_{a}^{b} w(t) f^{\gamma}(\sigma(t)) \Delta t\right) \mid \\
& \leqslant \frac{1}{m^{3}(a, b)} \int_{a}^{b} w(x)\left(\int_{a}^{b}\left|p_{w}(x, t)\right|\left|\left(f^{\gamma}(t)\right)^{\Delta}\right| \Delta t\right)\left(\int_{a}^{b}\left|p_{w}(x, t)\right|\left(g^{\gamma}(t)\right)^{\Delta} \mid \Delta t\right) \Delta x \\
& \leqslant \frac{M N}{m^{3}(a, b)} \int_{a}^{b} w(x)\left(\int_{a}^{b}\left|p_{w}(x, t)\right| \Delta t\right)^{2} \Delta x . \\
& \leqslant K \int_{a}^{b} \frac{w(x)}{m(a, b)}\left(\frac{1}{m(a, b)} \int_{a}^{b}\left|p_{w}(x, t)\right| \Delta t\right)^{2} \Delta x .
\end{aligned}
$$

Now, by using the definition of $\mathcal{H}(f, g)$, we get the desired inequality (3.1). The proof is complete.

Remark 3.2. By using the definition of $p_{w}(x, t)$, we see that

$$
\begin{aligned}
\int_{a}^{b}\left|p_{w}(x, t)\right| \Delta t & =\int_{a}^{x}\left|p_{w}(x, t)\right| \Delta t+\int_{x}^{b}\left|p_{w}(x, t)\right| \Delta t \\
& =\int_{a}^{x}\left|\left(\int_{a}^{t} w(\tau) \Delta \tau\right)\right| \Delta t+\int_{x}^{b}\left|\left(\int_{b}^{t} w(\tau) \Delta \tau\right)\right| \Delta t \\
& =\int_{a}^{x}\left(\int_{a}^{t} w(\tau) \Delta \tau\right) \Delta t-\int_{x}^{b}\left(\int_{b}^{t} w(\tau) \Delta \tau\right) \Delta t \\
& =x \int_{a}^{x} w(\tau) \Delta \tau-a \int_{a}^{a} w(\tau) \Delta \tau-\int_{a}^{x} w(t) \sigma(t) \Delta t-b \int_{b}^{b} w(\tau) \Delta \tau+x \int_{b}^{x} w(\tau) \Delta \tau+\int_{x}^{b} w(t) \sigma(t) \Delta t \\
& =x\left(\int_{a}^{x} w(\tau) \Delta \tau+\int_{b}^{x} w(\tau) \Delta \tau\right)-\left(\int_{a}^{x} w(t) \sigma(t) \Delta t-\int_{x}^{b} w(t) \sigma(t) \Delta t\right) \\
& =-x\left(\int_{a}^{x} \operatorname{sgn}(t-x) w(\tau) \Delta \tau+\int_{x}^{b} \operatorname{sgn}(t-x) w(\tau) \Delta \tau\right)
\end{aligned}
$$




$$
\begin{aligned}
& +\left(\int_{a}^{x} \operatorname{sgn}(t-x) w(t) \sigma(t) \Delta t+\int_{x}^{b} \operatorname{sgn}(t-x) w(t) \sigma(t) \Delta t\right) \\
= & -x \int_{a}^{b} \operatorname{sgn}(t-x) w(t) \Delta t+\int_{a}^{b} \operatorname{sgn}(t-x) w(t) \sigma(t) \Delta t \\
= & \int_{a}^{b} \operatorname{sgn}(t-x) w(t) \sigma(t) \Delta t-x \int_{a}^{b} \operatorname{sgn}(t-x) w(t) \Delta t \\
= & \int_{a}^{b}(\sigma(t)-x) w(t) \operatorname{sgn}(t-x) \Delta t .
\end{aligned}
$$

By combining the result in Remark 3.2 and Theorem 3.1, we get the following result.

Corollary 3.3. Assume that $\mathrm{M}=\sup _{\mathbf{a}<\mathrm{t}<\mathbf{b}}\left|\left(\mathrm{f}^{\gamma}(\mathrm{t})\right)^{\Delta}\right|<\infty$ and $\mathrm{N}=\sup _{\mathbf{a}<\mathrm{t}<\mathbf{b}}\left|\left(\mathrm{g}^{\gamma}(\mathrm{t})\right)^{\Delta}\right|<\infty$ and $\mathrm{K}=\mathrm{MN}>0$. Then we have

$$
|\mathcal{H}(\mathrm{f}, \mathrm{g})| \leqslant K \int_{a}^{b} \frac{w(x)}{m(a, b)}\left(\frac{1}{m(a, b)} \int_{a}^{b}(\sigma(t)-x) w(t) \operatorname{sgn}(t-x) \Delta t\right)^{2} \Delta x .
$$

If we assume that $f$ and $g$ are nondecreasing, we see that $f^{\gamma}(\sigma(t)) \geqslant f^{\gamma}(t)$ and $g^{\gamma}(\sigma(t)) \geqslant g^{\gamma}(t)$. This in addition to the assumptions of Theorem 3.1 give us the following result.

Corollary 3.4. Assume that $\mathrm{M}=\sup _{\mathbf{a}<\mathrm{t}<\mathrm{b}}\left|\left(\mathrm{f}^{\gamma}(\mathrm{t})\right)^{\Delta}\right|<\infty$ and $\mathrm{N}=\sup _{\mathrm{a}<\mathrm{t}<\mathrm{b}}\left|\left(\mathrm{g}^{\gamma}(\mathrm{t})\right)^{\Delta}\right|<\infty$, and $\mathrm{K}=\mathrm{MN}>0$. If $\mathrm{f}$ and $\mathrm{g}$ are nondecreasing, then we have

$$
\left|\mathcal{H}_{0}(f, g)\right| \leqslant K \int_{a}^{b} \frac{w(x)}{m(a, b)}\left(\frac{1}{m(a, b)} \int_{a}^{b}\left|p_{w}(x, t)\right| \Delta t\right)^{2} \Delta x,
$$

where

$$
\begin{aligned}
\mathcal{H}_{0}(\mathrm{f}, \mathrm{g})= & \frac{1}{\mathrm{~m}(\mathrm{a}, \mathrm{b})} \int_{a}^{b} w(\mathrm{t}) \mathrm{f}^{\gamma}(\mathrm{t}) g^{\gamma}(\mathrm{t}) \Delta \mathrm{t}-\frac{2}{m^{2}(\mathrm{a}, \mathrm{b})}\left(\int_{a}^{b} w(t) f^{\gamma}(\mathrm{t}) \Delta \mathrm{t}\right)\left(\int_{a}^{b} w(t) g^{\gamma}(\mathrm{t}) \Delta t\right) \\
& +\frac{1}{m^{3}(\mathrm{a}, \mathrm{b})}\left(\int_{a}^{b} w(t) g^{\gamma}(\sigma(\mathrm{t})) \Delta t\right)\left(\int_{a}^{b} w(t) f^{\gamma}(\sigma(t)) \Delta t\right) .
\end{aligned}
$$

Theorem 3.5. Assume that $M=\sup _{\mathbf{a}<\mathrm{t}<\mathbf{b}}\left|\left(f^{\gamma}(\mathrm{t})\right)^{\Delta}\right|<\infty$ and $\mathrm{N}=\sup _{\mathbf{a}<\mathrm{t}<\mathbf{b}}\left|\left(\mathrm{g}^{\gamma}(\mathrm{t})\right)^{\Delta}\right|<\infty$. Then we have

$$
\left|\mathcal{H}_{1}(f, g)\right| \leqslant \frac{1}{2 m(a, b)} \int_{a}^{b} w(x)\left[\left|g^{\gamma}(x)\right| M+\left|f^{\gamma}(x)\right| N\right]\left(\frac{1}{m(a, b)} \int_{a}^{b}\left|p_{w}(x, t)\right| \Delta t\right) \Delta x,
$$

where

$$
\begin{aligned}
\mathcal{H}_{1}(\mathrm{f}, \mathrm{g})= & \frac{1}{\mathrm{~m}(\mathrm{a}, \mathrm{b})} \int_{a}^{b} w(t) f^{\gamma}(\mathrm{t}) g^{\gamma}(\mathrm{t}) \Delta \mathrm{t} \\
& -\frac{1}{2}\left(\frac{1}{\mathrm{~m}(\mathrm{a}, \mathrm{b})} \int_{a}^{b} w(t) g^{\gamma}(\mathrm{t}) \Delta t\right)\left(\frac{1}{m(a, b)} \int_{a}^{b} w(t) f^{\gamma}(\sigma(t)) \Delta t\right) \\
& -\frac{1}{2}\left(\frac{1}{m(a, b)} \int_{a}^{b} w(t) f^{\gamma}(t) \Delta t\right)\left(\frac{1}{m(a, b)} \int_{a}^{b} w(t) g^{\gamma}(\sigma(t)) \Delta t\right) .
\end{aligned}
$$


Proof. Multiplying (3.2) by $\mathrm{g}^{\gamma}(\mathrm{x})$ and (3.3) by $\mathrm{f}^{\gamma}(\mathrm{x})$, adding the resulting identities and multiplying the final result with $w(x) /(2 m(a, b))$ and integrating over $[a, b]_{\mathbb{T}}$, we have

$$
\begin{aligned}
& \frac{1}{m(a, b)} \int_{a}^{b} w(t) f^{\gamma}(t) g^{\gamma}(t) \Delta t-\frac{1}{2 m^{2}(a, b)}\left(\int_{a}^{b} w(t) g^{\gamma}(t) \Delta t\right)\left(\int_{a}^{b} w(t) f^{\gamma}(\sigma(t)) \Delta t\right) \\
& \quad-\frac{1}{2 m^{2}(a, b)}\left(\int_{a}^{b} w(t) f^{\gamma}(t) \Delta t\right)\left(\int_{a}^{b} w(t) g^{\gamma}(\sigma(t)) \Delta t\right) \\
& =\frac{1}{2 m^{2}(a, b)} \int_{a}^{b} w(x)\left[g^{\gamma}(x)\left(\int_{a}^{b} p_{w}(x, t)\left(f^{\gamma}(t)\right)^{\Delta} \Delta t\right)\right. \\
& \left.+f^{\gamma}(x)\left(\int_{a}^{b} p_{w}(x, t)\left(g^{\gamma}(t)\right)^{\Delta} \Delta t\right) \Delta x\right],
\end{aligned}
$$

and taking absolute values then, we get

$$
\begin{aligned}
& \mid \frac{1}{m(a, b)} \int_{a}^{b} w(t) f^{\gamma}(t) g^{\gamma}(t) \Delta t-\frac{1}{2 m^{2}(a, b)}\left(\int_{a}^{b} w(t) g^{\gamma}(t) \Delta t\right)\left(\int_{a}^{b} w(t) f^{\gamma}(\sigma(t)) \Delta t\right) \\
& \quad-\frac{1}{2 m^{2}(a, b)}\left(\int_{a}^{b} w(t) f^{\gamma}(t) \Delta t\right)\left(\int_{a}^{b} w(t) g^{\gamma}(\sigma(t)) \Delta t\right) \mid \\
& \leqslant \frac{1}{2 m^{2}(a, b)} \int_{a}^{b} w(x)\left[\left|g^{\gamma}(x)\right|\left(\int_{a}^{b}\left|p_{w}(x, t)\right|\left|\left(f^{\gamma}(t)\right)^{\Delta}\right| \Delta t\right)+\left|f^{\gamma}(x)\right|\left(\int_{a}^{b}\left|p_{w}(x, t)\right|\left|\left(g^{\gamma}(t)\right)^{\Delta}\right| \Delta t\right)\right] \Delta x \\
& \leqslant \frac{1}{2 m(a, b)} \int_{a}^{b} w(x)\left[\left|g^{\gamma}(x)\right| M+\left|f^{\gamma}(x)\right| N\right]\left(\frac{1}{m(a, b)} \int_{a}^{b}\left|p_{w}(x, t)\right| \Delta t\right) \Delta x .
\end{aligned}
$$

Now, by using the definition of $\mathcal{H}_{1}(f, g)$, we get the desired inequality (3.5). The proof is complete.

By combining the result in Remark 3.2 and Theorem 3.5, we get the following result.

Corollary 3.6. Assume that $\mathrm{M}=\sup _{\mathbf{a}<\mathrm{t}<\mathrm{b}}\left|\left(\mathrm{f}^{\gamma}(\mathrm{t})\right)^{\Delta}\right|<\infty$ and $\mathrm{N}=\sup _{\mathrm{a}<\mathrm{t}<\mathrm{b}}\left|\left(\mathrm{g}^{\gamma}(\mathrm{t})\right)^{\Delta}\right|<\infty$. Then we have

$$
\left|\mathcal{H}_{1}(\mathrm{f}, \mathrm{g})\right| \leqslant \frac{1}{2 \mathrm{~m}(\mathrm{a}, \mathrm{b})} \int_{\mathrm{a}}^{\mathrm{b}} w(\mathrm{x})\left[\mathrm{g}^{\gamma}(\mathrm{x})|\mathrm{M}+| \mathrm{f}^{\gamma}(\mathrm{x}) \mid \mathrm{N}\right]\left(\frac{1}{\mathrm{~m}(\mathrm{a}, \mathrm{b})} \int_{\mathrm{a}}^{\mathrm{b}}(\sigma(\mathrm{t})-\mathrm{x}) w(\mathrm{t}) \operatorname{sgn}(\mathrm{t}-\mathrm{x}) \Delta \mathrm{t}\right) \Delta \mathrm{x} .
$$

If we assume that $f$ and $g$ are nondecreasing, we see that $f^{\gamma}(\sigma(t)) \geqslant f^{\gamma}(t)$ and $g^{\gamma}(\sigma(t)) \geqslant g^{\gamma}(t)$. This in addition to the assumptions of Theorem 3.5 gives us the following result.

Corollary 3.7. Assume that $M=\sup _{\mathbf{a}<\mathrm{t}<\mathrm{b}}\left|\left(\mathrm{f}^{\gamma}(\mathrm{t})\right)^{\Delta}\right|<\infty$ and $\mathrm{N}=\sup _{\mathrm{a}<\mathrm{t}<\mathrm{b}}\left|\left(\mathrm{g}^{\gamma}(\mathrm{t})\right)^{\Delta}\right|<\infty$. If $\mathrm{f}$ and $\mathrm{g}$ is nondecreasing, then we have

$$
\left|\mathcal{H}_{2}(f, g)\right| \leqslant \frac{1}{2 m(a, b)} \int_{a}^{b} w(x)\left[\left|g^{\gamma}(x)\right| M+\left|f^{\gamma}(x)\right| N\right]\left(\frac{1}{m(a, b)} \int_{a}^{b}\left|p_{w}(x, t)\right| \Delta t\right) \Delta x,
$$

where

$$
\mathcal{H}_{2}(\mathrm{f}, \mathrm{g})=\frac{1}{\mathrm{~m}(\mathrm{a}, \mathrm{b})} \int_{a}^{b} w(t) f^{\gamma}(\mathrm{t}) g^{\gamma}(\mathrm{t}) \Delta \mathrm{t}-\left(\frac{1}{\mathrm{~m}(\mathrm{a}, \mathrm{b})} \int_{a}^{b} w(t) f^{\gamma}(\mathrm{t}) \Delta \mathrm{t}\right)\left(\frac{1}{\mathrm{~m}(\mathrm{a}, \mathrm{b})} \int_{a}^{b} w(t) g^{\gamma}(\mathrm{t}) \Delta \mathrm{t}\right) .
$$

Corollary 3.8. In the case $\gamma=1$ in Theorems 3.1 and 3.5, we obtain the result in [34, Theorem 3.1]. 
Corollary 3.9. In the case $\gamma=1$ and $\mathbb{T}=\mathbb{R}$ in Theorems 3.1 and 3.5, we obtain the result in [23, Theorem 1].

Corollary 3.10. In the case $\gamma=1$ and $\mathbb{T}=\mathbb{Z}$ in Theorems 3.1 and 3.5 we obtain the discrete result in [34, Corollary 3.2].

Corollary 3.11. In the case $\gamma=1$ and $\mathbb{T}=q^{\mathbb{N}} \cup\{0\}(q>1)$ in Theorems 3.1 and 3.5 , we obtain the results in [34, Corollary 3.3].

To prove the following theorem and for the sake of simplicity, we define the operator $\mathcal{M}(f, g)$ by

$$
\begin{aligned}
\mathcal{M}(f, g)= & \frac{1}{m(a, b)} \int_{a}^{b} w(t) f^{\gamma}(t) g^{\gamma}(t) \Delta t-\frac{1}{m^{2}(a, b)}\left(\int_{a}^{b} w(t) f^{\gamma}(t) \Delta t\right)\left(\int_{a}^{b} w(t) g^{\gamma}(\sigma(t)) \Delta t\right) \\
& -\frac{1}{m^{2}(a, b)}\left(\int_{a}^{b} w(t) g^{\gamma}(t) \Delta t\right)\left(\int_{a}^{b} w(t) f^{\gamma}(\sigma(t)) \Delta t\right) \\
& +\frac{1}{m^{3}(a, b)}\left(\int_{a}^{b} w(t) f^{\gamma}(\sigma(t)) \Delta t\right)\left(\int_{a}^{b} w(t) g^{\gamma}(\sigma(t)) \Delta t\right) .
\end{aligned}
$$

Theorem 3.12. Assume that $M=\left\|\left(f^{\gamma}\right)^{\Delta}\right\|_{p}<\infty$ and $N=\left\|\left(g^{\gamma}\right)^{\Delta}\right\|_{p}<\infty$ and $K=M N>0$. Then we have

$$
|\mathcal{M}(f, g)| \leqslant K \int_{a}^{b} \frac{w(x)}{m^{3}(a, b)}\left(\int_{a}^{b}\left|p_{w}(x, t)\right|^{q} \Delta t\right)^{\frac{2}{q}} \Delta x
$$

where $\mathcal{M}(\mathrm{f}, \mathrm{g})$ is the mean power operator defined by (3.7).

Proof. From (3.4), we have

$$
\begin{gathered}
f^{\gamma}(x) g^{\gamma}(x)-\frac{f^{\gamma}(x)}{m(a, b)} \int_{a}^{b} w(t) g^{\gamma}(\sigma(t)) \Delta t-\frac{g^{\gamma}(x)}{m(a, b)} \int_{a}^{b} w(t) f^{\gamma}(\sigma(t)) \Delta t \\
\quad+\frac{1}{m^{2}(a, b)}\left(\int_{a}^{b} w(t) f^{\gamma}(\sigma(t)) \Delta t\right)\left(\int_{a}^{b} w(t) g^{\gamma}(\sigma(t)) \Delta t\right) \\
=\frac{1}{m^{2}(a, b)}\left(\int_{a}^{b} p_{w}(x, t)\left(f^{\gamma}(t)\right)^{\Delta} \Delta t\right)\left(\int_{a}^{b} p_{w}(x, t)\left(g^{\gamma}(t)\right)^{\Delta} \Delta t\right) .
\end{gathered}
$$

Multiplying with $w(x) / m(a, b)$, integrating and taking absolute values and using Hölder's inequality on time scales, we have

$$
\begin{aligned}
& \mid \frac{1}{m(a, b)} \int_{a}^{b} w(t) f^{\gamma}(t) g^{\gamma}(t) \Delta t-\frac{1}{m^{2}(a, b)}\left(\int_{a}^{b} w(t) f^{\gamma}(t) \Delta t\right)\left(\int_{a}^{b} w(t) g^{\gamma}(\sigma(t)) \Delta t\right) \\
& \quad-\frac{1}{m^{2}(a, b)}\left(\int_{a}^{b} w(t) g^{\gamma}(t) \Delta t\right)\left(\int_{a}^{b} w(t) f^{\gamma}(\sigma(t)) \Delta t\right) \\
& \quad+\frac{1}{m^{3}(a, b)}\left(\int_{a}^{b} w(t) g^{\gamma}(\sigma(t)) \Delta t\right)\left(\int_{a}^{b} w(t) f^{\gamma}(\sigma(t)) \Delta t\right) \mid \\
& \leqslant \frac{1}{m^{3}(a, b)} \int_{a}^{b} w(x)\left(\int_{a}^{b}\left|p_{w}(x, t)\right|\left|\left(f^{\gamma}(t)\right)^{\Delta}\right| \Delta t\right)\left(\int_{a}^{b}\left|p_{w}(x, t)\right|\left|\left(g^{\gamma}(t)\right)^{\Delta}\right| \Delta t\right) \Delta x \\
& \leqslant \frac{\left\|\left(f^{\gamma}\right)^{\Delta}\right\|\left\|_{p}\right\|\left(g^{\gamma}\right)^{\Delta}\|\|_{p} \int_{a}^{b} w(x)\left(\int_{a}^{b}\left|p_{w}(x, t)\right|^{q} \Delta t\right)^{\frac{2}{q}} \Delta x .}{m^{3}(a, b)}
\end{aligned}
$$




$$
\begin{aligned}
& \leqslant \frac{M N}{m^{3}(a, b)} \int_{a}^{b} w(x)\left(\int_{a}^{b}\left|p_{w}(x, t)\right|^{q} \Delta t\right)^{\frac{2}{q}} \Delta x . \\
& \leqslant K \int_{a}^{b} \frac{w(x)}{m^{3}(a, b)}\left(\int_{a}^{b}\left|p_{w}(x, t)\right|^{q} \Delta t\right)^{\frac{2}{q}} \Delta x .
\end{aligned}
$$

Now, by using the definition of $\mathcal{M}(f, g)$, we get the desired inequality (3.8). The proof is complete.

Remark 3.13. By using the definition of $p_{w}(x, t)$, we see that

$$
\begin{aligned}
\left(\int_{a}^{b}\left|p_{w}(x, t)\right|^{q} \Delta t\right)^{\frac{1}{q}} & =\left(\int_{a}^{x}\left|p_{w}(x, t)\right|^{q} \Delta t+\int_{x}^{b}\left|p_{w}(x, t)\right|^{q} \Delta t\right)^{\frac{1}{q}} \\
& =\left(\int_{a}^{x}\left|\int_{a}^{t} w(u) \Delta u\right|^{q} \Delta t+\int_{x}^{b}\left|\int_{b}^{t} w(u) \Delta u\right|^{q} \Delta t\right)^{\frac{1}{q}} \\
& =\left(\int_{a}^{x}\left(\int_{a}^{t} w(u) \Delta u\right)^{q} \Delta t+\int_{x}^{b}\left(\int_{b}^{t} w(u) \Delta u\right)^{q} \Delta t\right)^{\frac{1}{q}} \\
& =\left(\int_{a}^{x} m^{q}(a, t) \Delta t+\int_{x}^{b} m^{q}(t, b) \Delta t\right)^{\frac{1}{q}} .
\end{aligned}
$$

By combining the result in Remark 3.13 and Theorem 3.12, we get the following result.

Corollary 3.14. Assume that $\mathrm{M}=\left\|\left(\mathrm{f}^{\gamma}\right)^{\Delta}\right\|_{\mathrm{p}}<\infty$ and $\mathrm{N}=\left\|\left(\mathrm{g}^{\gamma}\right)^{\Delta}\right\|_{\mathrm{p}}<\infty$ and $\mathrm{K}=\mathrm{MN}>0$. Then we have

$$
|\mathcal{M}(f, g)| \leqslant K \int_{a}^{b} \frac{w(x)}{m^{3}(a, b)}\left(\int_{a}^{x} m^{q}(a, t) \Delta t+\int_{x}^{b} m^{q}(t, b) \Delta t\right)^{\frac{2}{q}} \Delta x .
$$

If we assume that $f$ and $g$ are nondecreasing, we see that $f^{\gamma}(\sigma(t)) \geqslant f^{\gamma}(t)$ and $g^{\gamma}(\sigma(t)) \geqslant g^{\gamma}(t)$. This in addition to the assumptions of Theorem 3.12 gives us the following result.

Corollary 3.15. Assume that $\mathrm{M}=\left\|\left(\mathrm{f}^{\gamma}\right)^{\Delta}\right\|_{\mathrm{p}}<\infty$ and $\mathrm{N}=\left\|\left(\mathrm{g}^{\gamma}\right)^{\Delta}\right\|_{\mathrm{p}}<\infty$ and $\mathrm{K}=\mathrm{MN}>0$. If $\mathrm{f}$ and $\mathrm{g}$ are nondecreasing, then we have

$$
\left|\mathcal{M}_{0}(f, g)\right| \leqslant K \int_{a}^{b} \frac{w(x)}{m^{3}(a, b)}\left(\int_{a}^{b}\left|p_{w}(x, t)\right|^{q} \Delta t\right)^{\frac{2}{q}} \Delta x
$$

where

$$
\begin{aligned}
\mathcal{M}_{0}(f, g)= & \frac{1}{m(a, b)} \int_{a}^{b} w(t) f^{\gamma}(t) g^{\gamma}(t) \Delta t-\frac{2}{m^{2}(a, b)}\left(\int_{a}^{b} w(t) f^{\gamma}(t) \Delta t\right)\left(\int_{a}^{b} w(t) g^{\gamma}(t) \Delta t\right) \\
& +\frac{1}{m^{3}(a, b)}\left(\int_{a}^{b} w(t) g^{\gamma}(\sigma(t)) \Delta t\right)\left(\int_{a}^{b} w(t) f^{\gamma}(\sigma(t)) \Delta t\right) .
\end{aligned}
$$

Theorem 3.16. Assume that $\mathrm{M}=\left\|\left(\mathrm{f}^{\gamma}\right)^{\Delta}\right\|_{\mathrm{p}}<\infty$ and $\mathrm{N}=\left\|\left(\mathrm{g}^{\gamma}\right)^{\Delta}\right\|_{\mathrm{p}}<\infty$. Then we have

$$
\left|\mathcal{M}_{1}(f, g)\right| \leqslant \frac{1}{2 m^{2}(a, b)} \int_{a}^{b} w(x)\left[\left|g^{\gamma}(x)\right| M+\left|f^{\gamma}(x)\right| N\right]\left(\int_{a}^{b}\left|p_{w}(x, t)\right|^{q} \Delta t\right)^{\frac{1}{q}} \Delta x,
$$


where

$$
\begin{aligned}
\mathcal{M}_{1}(f, g)= & \frac{1}{m(a, b)} \int_{a}^{b} w(t) f^{\gamma}(t) g^{\gamma}(t) \Delta t-\frac{1}{2}\left(\frac{1}{m(a, b)} \int_{a}^{b} w(t) g^{\gamma}(t) \Delta t\right)\left(\frac{1}{m(a, b)} \int_{a}^{b} w(t) f^{\gamma}(\sigma(t)) \Delta t\right) \\
& -\frac{1}{2}\left(\frac{1}{m(a, b)} \int_{a}^{b} w(t) f^{\gamma}(t) \Delta t\right)\left(\frac{1}{m(a, b)} \int_{a}^{b} w(t) g^{\gamma}(\sigma(t)) \Delta t\right) .
\end{aligned}
$$

Proof. From (3.6), we have

$$
\begin{aligned}
& \frac{1}{m(a, b)} \int_{a}^{b} w(t) f^{\gamma}(t) g^{\gamma}(t) \Delta t-\frac{1}{2 m^{2}(a, b)}\left(\int_{a}^{b} w(t) g^{\gamma}(t) \Delta t\right)\left(\int_{a}^{b} w(t) f^{\gamma}(\sigma(t)) \Delta t\right) \\
& \quad-\frac{1}{2 m^{2}(a, b)}\left(\int_{a}^{b} w(t) f^{\gamma}(t) \Delta t\right)\left(\int_{a}^{b} w(t) g^{\gamma}(\sigma(t)) \Delta t\right) \\
& =\frac{1}{2 m^{2}(a, b)} \int_{a}^{b} w(x)\left[g^{\gamma}(x)\left(\int_{a}^{b} p_{w}(x, t)\left(f^{\gamma}(t)\right)^{\Delta} \Delta t\right)+f^{\gamma}(x)\left(\int_{a}^{b} p_{w}(x, t)\left(g^{\gamma}(t)\right)^{\Delta} \Delta t\right)\right] \Delta x .
\end{aligned}
$$

taking absolute values and using Hölder's inequality on time scales, we get

$$
\begin{aligned}
& \mid \frac{1}{m(a, b)} \int_{a}^{b} w(t) f^{\gamma}(t) g^{\gamma}(t) \Delta t-\frac{1}{2 m^{2}(a, b)}\left(\int_{a}^{b} w(t) g^{\gamma}(t) \Delta t\right)\left(\int_{a}^{b} w(t) f^{\gamma}(\sigma(t)) \Delta t\right) \\
& \quad-\frac{1}{2 m^{2}(a, b)}\left(\int_{a}^{b} w(t) f^{\gamma}(t) \Delta t\right)\left(\int_{a}^{b} w(t) g^{\gamma}(\sigma(t)) \Delta t\right) \mid \\
& \leqslant \frac{1}{2 m^{2}(a, b)} \int_{a}^{b} w(x)\left[\left|g^{\gamma}(x)\right|\left(\int_{a}^{b}\left|p_{w}(x, t)\right|\left|\left(f^{\gamma}(t)\right)^{\Delta}\right| \Delta t\right)+\left|f^{\gamma}(x)\right|\left(\int_{a}^{b}\left|p_{w}(x, t)\right|\left|\left(g^{\gamma}(t)\right)^{\Delta}\right| \Delta t\right)\right] \Delta x \\
& \leqslant \frac{1}{2 m^{2}(a, b)} \int_{a}^{b} w(x)\left[\left|g^{\gamma}(x)\right|\left|\left(f^{\gamma}\right)^{\Delta}\left\|_{p}\left(\int_{a}^{b}\left|p_{w}(x, t)\right|^{q} \Delta t\right)^{\frac{1}{q}}+\left|f^{\gamma}(x)\right|\right\|\left(g^{\gamma}\right)^{\Delta} \|_{p}\left(\int_{a}^{b}\left|p_{w}(x, t)\right|^{q} \Delta t\right)^{\frac{1}{q}}\right] \Delta x\right. \\
& \leqslant \frac{1}{2 m^{2}(a, b)} \int_{a}^{b} w(x)\left[\left|g^{\gamma}(x)\right| M+\left|f^{\gamma}(x)\right| N\right] \times\left(\int_{a}^{b}\left|p_{w}(x, t)\right|^{q} \Delta t\right)^{\frac{1}{q}} \Delta x .
\end{aligned}
$$

Now, by using the definition of $\mathcal{M}_{1}(f, g)$, we get the desired inequality (3.9). The proof is complete.

By combining the result in Remark 3.13 and Theorem 3.16, we get the following result.

Corollary 3.17. Assume that $M=\left\|\left(f^{\gamma}\right)^{\Delta}\right\|_{p}<\infty$ and $N=\left\|\left(g^{\gamma}\right)^{\Delta}\right\|_{p}<\infty$. Then we have

$$
\left|\mathcal{M}_{1}(f, g)\right| \leqslant \frac{1}{2 m^{2}(a, b)} \int_{a}^{b} w(x)\left[\left|g^{\gamma}(x)\right| M+\left|f^{\gamma}(x)\right| N\right]\left(\int_{a}^{x} m^{q}(a, t) \Delta t+\int_{x}^{b} m^{q}(t, b) \Delta t\right)^{\frac{1}{q}} \Delta x .
$$

If we assume that $f$ and $g$ are nondecreasing, we see that $f^{\gamma}(\sigma(t)) \geqslant f^{\gamma}(t)$ and $g^{\gamma}(\sigma(t)) \geqslant g^{\gamma}(t)$. This in addition to the assumptions of Theorem 3.16 gives us the following result.

Corollary 3.18. Assume that $\mathrm{M}=\left\|\left(\mathrm{f}^{\gamma}\right)^{\Delta}\right\|_{\mathrm{p}}<\infty$ and $\mathrm{N}=\left\|\left(\mathrm{g}^{\gamma}\right)^{\Delta}\right\|_{\mathrm{p}}<\infty$. If $\mathrm{f}$ and $\mathrm{g}$ is nondecreasing, then we have

$$
\left|\mathcal{M}_{2}(f, g)\right| \leqslant \frac{1}{2 m^{2}(a, b)} \int_{a}^{b} w(x)\left[\left|g^{\gamma}(x)\right| M+\left|f^{\gamma}(x)\right| N\right]\left(\int_{a}^{b}\left|p_{w}(x, t)\right|^{q} \Delta t\right)^{\frac{1}{q}} \Delta x
$$

where

$$
\mathcal{M}_{2}(f, g)=\frac{1}{m(a, b)} \int_{a}^{b} w(t) f^{\gamma}(t) g^{\gamma}(t) \Delta t-\left(\frac{1}{m(a, b)} \int_{a}^{b} w(t) f^{\gamma}(t) \Delta t\right)\left(\frac{1}{m(a, b)} \int_{a}^{b} w(t) g^{\gamma}(t) \Delta t\right) .
$$


Corollary 3.19. In the case $\gamma=1$ in Theorems 3.12 and 3.16, we obtain Theorem 3.3 in [34].

Corollary 3.20. In the case $\gamma=1$ and $\mathbb{T}=\mathbb{R}$ in Theorems 3.12 and 3.16 we obtain Theorem 3 in [4].

Corollary 3.21. In the case $\gamma=1$ and $\mathbb{T}=\mathbb{Z}$ in Theorems 3.12 and 3.16 we obtain the discrete inequality proved in [34, Corollary 3.8].

Corollary 3.22. In the case $\gamma=1$ and $\mathbb{T}=q^{\mathbb{Z}} \cup\{0\}(q>1)$ in Theorems 3.12 and 3.16, we obtain Corollary 3.9 in [34].

\section{References}

[1] R. P. Agarwal, M. Bohner, A. Peterson, Inequalities on time scales: a survey, Math. Inequal. Appl., 4 (2001), $535-557$. 1

[2] R. P. Agarwal, D. O’Regan, S. H. Saker, Dynamic Inequalities on Time Scales, Springer, Cham, (2014). 1

[3] R. P. Agarwal, D. O’Regan, S. H. Saker, Hardy Type Inequalities on Time Scales, Springer, Cham, (2016). 1

[4] F. Ahmad, N. A. Mir, A. Rafiq, Weighted ČebyŠev type inequalities involving functions whose first derivatives belong to $\mathrm{L}_{\mathrm{p}}(\mathrm{a}, \mathrm{b}),(1 \leqslant \mathrm{p} \leqslant \infty)$, Kragujevac J. Math., 32 (2009), 13-26. 1, 3.20

[5] G. A. Anastassiou, Duality principle of time scales and inequalities, Appl. Anal., 89 (2010), 1837-1854. 1

[6] N. S. Barnett, S. S. Dragomir, An inequality of Ostrowski's type for cumulative distribution functions, Kyungpook Math. J., 39 (1999), 303-311. 1

[7] N. S. Barnett, S. S. Dragomir, An Ostrowski type inequality for a random variable whose probability density function belongs to $\mathrm{L}_{\infty}[\mathrm{a}, \mathrm{b}]$, Nonlinear Anal. Forum, 5 (2000), 125-135. 1

[8] M. Bohner, T. S. Hassan, T. X. Li, Fite-Hille-Wintner-type oscillation criteria for second-order half-linear dynamic equation with deviating arguments, Indag. Math. (N.S.), 29 (2018), 548-560. 1

[9] M. Bohner, T. X. Li, Kamenev-type criteria for nonlinear damped dynamic equations, Sci. China Math., 58 (2015), 14451452. 1

[10] M. Bohner, T. Matthews, The Grüss inequality on time scales, Commun. Math. Anal., 3 (2007), 1-8. 1

[11] M. Bohner, T. Matthews, Ostrowski inequalities on time scales, JIPAM. J. Inequal. Pure Appl. Math., 9 (2008), 8 pages. 1

[12] M. Bohner, T. Matthews, A. Tuna, Weighted Ostrowski-Grüss inequalities on time scales, Afr. Diaspora J. Math., 12 (2011), 89-99. 1

[13] M. Bohner, A. Peterson, Dynamic Equations on Time Scales, An Introduction with Applications, Birkhäuser, Boston, (2001). 2, 2

[14] M. Bohner, A. Peterson, Advances in Dynamic Equations on Time Scales, Birkhäuser Boston, Boston, (2003). 2, 2

[15] P. L. ČebyŠev, Sur les expressions approximatives des integrales definies par les autres prises entre les memes limites, Proc. Math. Soc. Charkov, 2 (1882), 93-98. 1

[16] S. S. Dragomir, P. Cerone, J. Roumeliotis, A new generalization of Ostrowskis intergral inequality for mappings whose derivatives are bounded and applications in numerical integration and for special means, Appl. Math. Lett., 13 (2000), 19-25. 1

[17] G. Grüss, Über das Maximum des absoluten Betrages von $\frac{1}{(b-a)} \int_{a}^{b} f(x) g(x) d x-\frac{1}{(b-a)^{2}} \int_{a}^{b} f(x) d x \int_{a}^{b} g(x) d x, M a t h$. Z., 39 (1935), 215-226. 1

[18] S. Hilger, Ein Maßkettenkalkül mit Anwendung auf Zentrumsmannigfaltigkeiten, Ph. D. Thesis, Universität Würzburg, (1988). 1

[19] S. Hilger, Analysis on measure chains-A unified approach to continuous and discrete calculus, Result Math., 18 (1990), 18-56.

[20] Z. A. Karian, E. J. Dudewicz, Fitting Statistical Distributions, CRC Press, Boca Raton, (2000). 1

[21] A. Ostrowski, Über die Absolutabweichung einer differentiierbaren Funktion von ihrem Integralmittelwert, Comment. Math. Helv., 10 (1937), 226-227. 1

[22] B. G. Pachpatte, On a new generalization of Ostrowski type inequality, Tamkang J. Math., 38 (2007), 335-339. 1

[23] A. Rafiq, N. A. Mir, F. Ahmad, Weighted ČebyŠev-Ostrowski type inequalities, Appl. Math. Mech. (English Ed.), 28 (2007), 901-906. 1, 3.9

[24] S. H. Saker, Hardy-Leindler type inequalities on time scales, Appl. Math. Inf. Sci., 8 (2014), 2975-2981. 1

[25] S. H. Saker, R. R. Mahmoud, M. M. Osman, R. P. Agarwal, Some new generalized forms of Hardy's type inequality on time scales, Math. Inequal. Appl., 20 (2017), 459-481.

[26] S. H. Saker, R. R. Mahmoud, A. Peterson, Some Bennett-Copson type inequalities on time scales, J. Math. Inequal., 10 (2016), 471-489.

[27] S. H. Saker, R. R. Mahmoud, A. Peterson, Weighted Hardy-type inequalities on time scales with Applications, Mediter. J. Math., 13 (2016), 585-606.

[28] S. H. Saker, D. O’Regan, Hardy and Littlewood inequalities on time scales, Bull. Malays. Math. Sci. Soc., 39 (2016), 527-543. 
[29] S. H. Saker, D. O’Regan, R. P. Agarwal, Generalized Hardy, Copson, Leindler and Bennett inequalities on time scales, Math. Nachr., 287 (2014), 686-698.

[30] S. H. Saker, M. M. Osman, D. O'Regan, R. P. Agarwal, Some new Opial dynamic inequalities with weighted functions on time scales, Math. Inequal. Appl., 18 (2015), 1171-1187.

[31] S. H. Saker, M. M. Osman, D. O'Regan, R. P. Agarwal, Inequalities of Hardy-type and generalizations on time scales, Analysis (Berlin), 38 (2018), 47-62.

[32] S. H. Saker, M. M. Osman, D. O’Regan, R. P. Agarwal, Lyapunov inequalities for dynamic equations via new Opial type inequalities, Hacettepe J. Math. Stat., 47 (2018), 1544-1558.

[33] S. H. Saker, M. M. Osman, D. O’Regan, R. P. Agarwal, Levinson type inequalities and their extensions via convexity on time scales, Rev. R. Acad. Cienc. Exactas Fís. Nat. Ser. A Mat. RACSAM, 113 (2019), 299-314. 1

[34] A. Tuna, W. J. Liu, New weighted ČebyŠev-Ostrowski type integral inequalities on time scales, J. Math. Inequal., 10 (2016), 327-356. 1, 3.8, 3.10, 3.11, 3.19, 3.21, 3.22 